Check for updates

Cite this: Mater. Adv., 2021, 2,3264

Received 20th February 2021 Accepted 14th April 2021

DOI: $10.1039 / \mathrm{d} 1 \mathrm{ma00153a}$

rsc.li/materials-advances

\section{Translation of a chemical reaction to electrical signal generation: an organic-inorganic complexation strategy $\dagger$}

\author{
Venkata K Perla, Sarit K Ghosh and Kaushik Mallick (D) *
}

A complexation strategy involving the addition of cysteine to a $\mathrm{Cu}(\mathrm{I})$-polyaniline composite system, where cysteine performed the role of a ligand, has been reported. The in situ electrical measurement of the complexation process exhibits a resistive switching behaviour with an ON-to-OFF ratio of $5 \times 10^{2}$ and follows the Ohmic and PooleFrenkel emission mechanism for the low-conductance state and Ohmic behaviour for the high-conductance state, with a volatile resistive switching property. Further, the polyaniline-stabilized copper sulfide nanoparticle-based device (where cysteine acts as the sulphur source) exhibits a bipolar resistive switching behaviour, where the low conductance state follows the Poole-Frenkel emission mechanism and the Ohmic mechanism was observed for the high conductance state. The device exhibits excellent endurance and retention capacity for $2 \times 10^{3}$ cycles with an average ON-to-OFF ratio of $10^{4}$, with a nonvolatile resistive switching behaviour.

The interfacing of electronics with biology is significantly important as the soft nature of the biologically relevant organic molecules offers a range of benefits, including mechanical flexibility and compatibility with the physiological system. ${ }^{1} \mathrm{~A}$ substantial amount of research studies have been reported, where the biomolecules are proposed as computing elements. ${ }^{2,3}$ Some particular type of redox proteins demonstrated as electronic materials and functioned as electron-transport junctions. ${ }^{4,5}$

A protein detection and quantification technique is applied for therapeutics, clinical diagnosis and treatment as well as for research on the cell signalling technology. There are different methods that are available in literature to detect protein molecules in different organisms. The enzyme-free colorimetric detection of $\alpha$-fetoprotein has been reported by organic molecule-modified quantum dots or metal-organic frameworks through a signal generation and amplification technique. ${ }^{6,7}$ The ferritin-based redox protein displayed a memristive

Department of Chemical Sciences, University of Johannesburg, P.O. Box: 524, Auckland Park, 2006, South Africa.E-mail: kaushikm@uj.ac.za

$\dagger$ Electronic supplementary information (ESI) available. See DOI: 10.1039/ d1ma00153a behaviour, where the hysteretic resistive switching effect was reported due to the electrochemical processes occurring in the active centre of ferritin, $\mathrm{Fe}(\mathrm{II}) / \mathrm{Fe}$ (III). ${ }^{8}$ In the field of biomolecular electronics, Azurin, a bacterial blue copper protein, stood out as one of the most widely studied proteins, where $\mathrm{Cu}$ ions performed the key function as an electron transfer agent. ${ }^{9}$ Copper ions play a crucial role in the physiological system, and the interaction study between copper and organic ligands, with biological relevance, is considered as one of the most fertile pitches in multidisciplinary research platform. Copper, a redoxactive element, in the form of zero-valent, uni-valent and bi-valent, not only serves the role of a catalyst ${ }^{10-13}$ but also acts as a structural co-factor for a wide range of biomolecules. ${ }^{14}$ Among numerous biomolecules, protein molecules have attracted tremendous attention for the integration of multifunctional molecular devices. ${ }^{15}$ On the other hand, amino acid such as cysteine with a thiol-side chain often participates in enzymatic reactions as a nucleophilic agent.

The cysteine-copper complexes perform numerous functions in the biological, environmental, and medical fields. ${ }^{16}$ The formation constants of Cu-complexes with cysteine based on the thermodynamic model confirmed that a univalent copperion is predominant in blood plasma, aqueous humour and cornea of the human eye. ${ }^{17}$ The $\mathrm{Cu}(\mathrm{I})$-cysteine complex also acts as a hole scavenging co-catalyst that influences the activity of CdSe for hydrogen production in the presence of visible light and also minimizes the electron-hole pair recombination due to photo-excitation. $^{18}$

The translation of a chemical reaction to electrical signal generation is a well-established method in electrochemical sensing applications. ${ }^{13,19,20}$ In this communication, we reported a typical complexation reaction between $\mathrm{Cu}(\mathrm{I})$ and cysteine, and the chemical reaction was in situ translated in the form of an electrical signal that exhibited a resistive switching effect. We extrapolated the conventional sensing method by fabricating a metal-(active material)-metal type of device and studied the transport mechanism of the reaction between $\mathrm{Cu}(\mathrm{I})$ and cysteine. The reactant $\mathrm{Cu}(\mathrm{I})$ was synthesized using a 


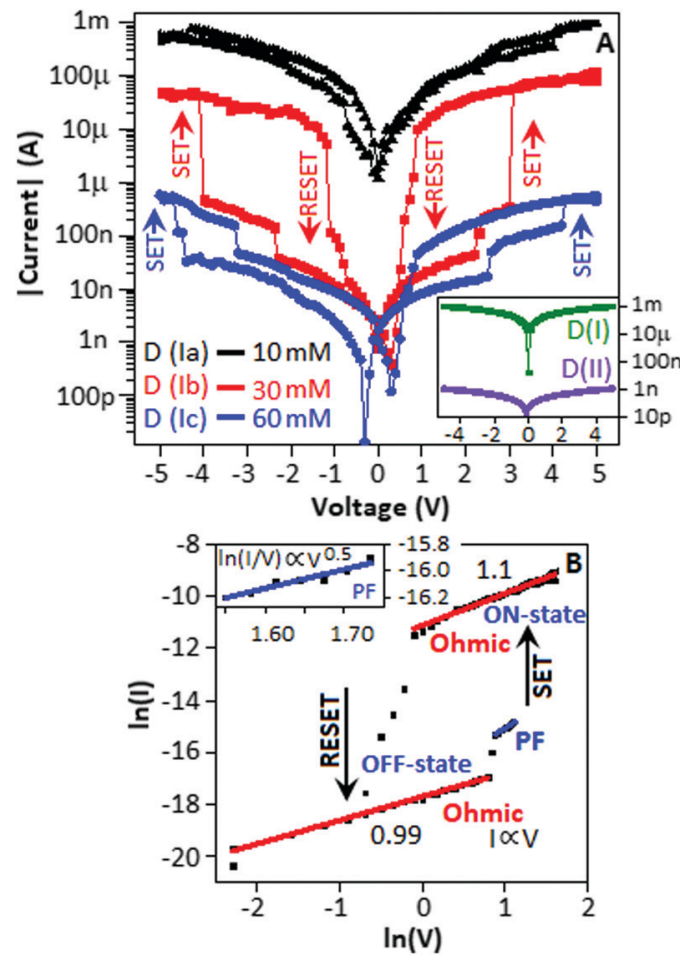

Fig. 1 (A) The current-voltage (semi-log) behaviour of the devices, D (la), $D(\mathrm{Ib})$ and $D(\mathrm{IC})$, after the addition of $5 \mu \mathrm{L}$ of cysteine solution at concentrations of 10,30 and $60 \mathrm{mM}$, respectively. The inset figure represents the $I-V$ behaviour of $D$ (I) and $D$ (II). (B) The transport mechanism of $D(\mathrm{Ib})$ was fitted with the Ohmic and PF for the OFF-state and Ohmic for the ON-state (inset: The graphical representation of the PF region).

previously reported method in the form of $\mathrm{Cu}(\mathrm{I})$-polyaniline (CuP). ${ }^{21-23}$

We studied the electrical property of the cysteine (Cys) and $\mathrm{Cu}(\mathrm{I})$ complexation reaction in the form of a device. The detail fabrication method is available in Experimental Section. Fig. 1A (inset) shows the graphical representation (semi-log) of the current-voltage $(I-V)$ behaviour of $\mathrm{Cu}(\mathrm{I})$-polyaniline (Au-CuP-Au), $\mathrm{D}$ (I) and cysteine (Au-Cys-Au), D (II) based device when the voltage sweeps are applied from 0 to $+5 \mathrm{~V},+5$ to $-5 \mathrm{~V}$ and -5 to $0 \mathrm{~V}$ directions. Fig. 1A (main panel) shows the $I-V$ characteristic, under the abovementioned sweep conditions, when $5 \mu \mathrm{L}$ of cysteine solution at concentrations of 10,30 and $60 \mathrm{mM}$ are added individually into the three sets of devices D (I) in the form of droplets at the boundary position of the top electrodes. They are represented as D (Ia), D (Ib) and D (Ic), respectively.

The change in the $I-V$ behaviour indicated that the complexation process occurred between $\mathrm{Cu}(\mathrm{I})$ and cysteine. The formation of $\mathrm{Cu}(\mathrm{I})$ complexes with cysteine was well-documented in literature. ${ }^{16,17,24}$ In the case of $\mathrm{D}(\mathrm{Ia})$, the $I-V$ characteristic exhibited a deviation from D (I) but without a proper resistive switching, while the devices D (Ib) and D (Ic) exhibited a resistive switching with an ON-to-OFF ratio of $5 \times 10^{-2}$ and $\sim 10^{1}$, respectively. In the case of devices D (Ia) and $\mathrm{D}$ (Ic), the conductivity values were close to the devices made with CuP, D (I), and cysteine, D (II), respectively, due to the incorporation of low and high concentrations of cysteine into the device D (I). For rest of our study, we considered the current-voltage characteristic of the device, $\mathrm{D}(\mathrm{Ib})$, where the maximum ON-OFF ratio of $\left(5 \times 10^{-2}\right)$ was achieved.

The device, D (Ib), exhibited two SET processes (the process of transferring the device from a low conductance to a high conductance state) at 2.2 and $3.0 \mathrm{~V}$ during the sweep direction from 0 to $+5 \mathrm{~V}$, and the RESET process (the process of transferring the device from a high conductance to a low conductance state) was observed at $0.9 \mathrm{~V}$ during the sweep direction from +5 to $-5 \mathrm{~V}$. Similarly, the device exhibited two SET processes at -2.3 and $-4.0 \mathrm{~V}$ and a RESET process at $-1.1 \mathrm{~V}$ on the other side of the $I-V$ plot. The entire $I-V$ plot demonstrated a unipolar behaviour with a symmetric ' $S$ ' type nature. ${ }^{25,26}$

During the voltage sweep from $0 \mathrm{~V}$ to $5 \mathrm{~V}$, a steady improvement in the current value was observed within the voltage range from 0 to $2.2 \mathrm{~V}$. At $2.2 \mathrm{~V}$, a sudden improvement in the current value can be explained in terms of an establishment of a transition state that resulted in the increase in the reduction potential value of $\mathrm{Cu}(\mathrm{I}){ }^{27}$ A further increase in the voltage influenced the reduction of $\mathrm{Cu}(\mathrm{I})$ with the formation of atomic copper, and at a potential value of $3.0 \mathrm{~V}$, when adequate numbers of atomic copper were formed, the device exhibited a complete SET process. For a voltage sweep from $+5 \mathrm{~V}$ to $-5 \mathrm{~V}$, a steady decrease in the current value was observed within the voltage range from +5 to $0.9 \mathrm{~V}$, which is presumably due to the slow oxidation of the copper atom. A subsequent sharp current fall (RESET) at $0.9 \mathrm{~V}$ indicated that the majority of copper atoms were oxidized and converted to $\mathrm{Cu}(\mathrm{I})$. The left-hand side of the $I-V$ plot also validated a similar mechanism. The currentvoltage characteristic of the device, D (Ib), followed an Ohmic mechanism within a voltage range from 0 to $2.2 \mathrm{~V}$ (OFF-state) (Fig. 1B). Initially, a small number of electrons were generated at the electrode-[Cu(I)-CYS] interface, and under the influence of a potential, the mobility of the electrons was attributed to the Ohmic conduction, where the current was proportional to the electric field with a slope value of $0.99 .^{28}$ As mentioned previously, a further increase in voltage (from 2.2 to $3.0 \mathrm{~V}$ ) influenced the reduction of $\mathrm{Cu}(\mathrm{I})$ with the formation of an atomic copper, followed by the copper nanoparticles. For a voltage ranging from 2.2 to $3.0 \mathrm{~V}$ (OFF-state) (Fig. 1B (inset)), the electrons could move slowly through the matrix and be trapped on copper nanoparticles. With an increase in the electric field, the electrons migrated from its localized state and moved to a conduction band, and the transport behaviour was followed by the Poole-Frenkel emission https://en.wikipedia.org/wiki/Poole\%E2\%80\%93Frenkel_effect-cite_ note-Sze-1 mechanism, $\ln (I / V) \alpha V^{0.5} \cdot{ }^{29}$ The presence of such type of two conduction mechanisms dominating at different bias voltage regions was reported earlier at a low conductance state. A highly oriented $\mathrm{ZnO}$ thin film exhibited an Ohmic behaviour and a PooleFrenkel emission at low- and high field regions for a low conductance state. ${ }^{30}$ In a similar fashion, a thin film of the $\mathrm{Gd}: \mathrm{SiO}_{2}$-based device under an illumination condition exhibited an Ohmic behaviour at a low-field region and a Poole-Frenkel emission at a high electric field region for the low conductance state. ${ }^{31}$

The formation of two SET-type characteristics at 2.2 and $-2.4 \mathrm{~V}$ (Fig. 1A, within the box) in both sides of the current-voltage graph 
was presumably due to the initialization process of atomic copper formation. For the ON-state, within a potential range from +5 to $0.9 \mathrm{~V}$, the transport mechanism was influenced by an Ohmic behaviour with a slope value of 1.10 .

The memory behaviour of a device mainly depends on the endurance and rewritable properties. For the endurance study, $\mathrm{a}-2 \mathrm{~V}$ pulse train (50\% duty cycle) is employed on the device $\mathrm{D}$ (Ib), as shown in Fig. 2A (inset). The device exhibited a stable OFF-state within the experimented cycle number of $2 \times 10^{3}$, whereas the ON-state demonstrated a dilapidation after $10^{3}$ cycles, and the device appeared volatile in nature. To investigate the ability of the device in differentiating the low conductance state $\left(R_{0}\right)$ from the high conductance state $\left(R_{1}\right)$ after the erasing and writing processes, respectively, the Write-Read (1)-EraseRead (0) $\left(\mathrm{WR}_{1} \mathrm{ER}_{0}\right)$ cycle was designed (ESI, $\dagger$ Fig. S1). The device $\mathrm{D}(\mathrm{I})$ was subjected to the $\mathrm{W} R_{1} \mathrm{E} R_{0}$ cycle, and it showed no difference between the $R_{1}$ and $R_{0}$ states in terms of the current value (Fig. 2B, zone (a)). With the addition of cysteine $(5 \mu \mathrm{L}$ of $30 \mathrm{mM}$ ), after certain buffer period (Fig. 2B, buffer zone), the device demonstrated a current difference value of $6 \mu \mathrm{A}$ between $R_{1}$ and $R_{0}$ states (Fig. 2B, zone (b)), which indicated that the complex system had the ability to identify Read (1) and Read (0)-states, clearly.

In a separate experiment, we synthesized polyanilinestabilized copper sulfide nanoparticles (PACS) using cysteine as the sulphur source (detailed synthesis protocol is available in Experimental section). The fabrication of polyaniline-stabilized copper sulfide nanoparticles is a two-step process. The first step involved the formation of $\mathrm{CuP}$ by a reaction between copper sulphate and aniline, and the second step was the complexation between $\mathrm{Cu}(\mathrm{I})$-polyaniline and cysteine. The second step is a slow process that involved the complexation and formation of a transition state that ultimately formed copper sulfide nanoparticles encapsulated within the polyaniline matrix. The synthesis of metal sulfide using cysteine as the sulphur source is welldocumented in the literature. ${ }^{32,33}$ The PACS-based organicinorganic hybrid system was used as an active material for resistive random-access memory applications. The detailed device fabrication method is available in Experimental section.

The device made with PACS (Au-PACS-Au) was subjected to the voltage sweep from 0 to $+5 \mathrm{~V},+5$ to $-5 \mathrm{~V}$ and -5 to $0 \mathrm{~V}$ directions, and the current response is plotted in the Fig. 3A. During the voltage sweep from 0 to $+5 \mathrm{~V}$, the device exhibited a SET process at $3.8 \mathrm{~V}$, whereas the RESET phenomena were observed at $-3.3 \mathrm{~V}$ during the voltage sweep direction from +5 to $-5 \mathrm{~V}$. The device showed a stable OFF-state behaviour during a voltage sweep from -5 to $0 \mathrm{~V}$. The current-voltage characteristic of the device exhibited a bipolar resistive switching behaviour with an asymmetric ' $S$ ' type character. ${ }^{25}$ To realize the transport property of the device, the current-voltage graph was fitted with different theoretical models for both the low conducting (OFF) and high conducting (ON) states. Fig. 3B exhibits the $I-V$ characteristic, where the low conductance state follows the Poole-Frenkel (PF) emission mechanism, $\ln (I / V) \propto \sqrt{V},{ }^{34}$ and the high conductance state is fitted with the Ohmic conduction mechanism, $\ln (I) \propto \ln (V)$, with a slope of $1.14 .^{35}$

Initially, the electrons were trapped and localized on the polyaniline matrix, and under a suitable potential, the trapped electrons were released and passed through various densities of states of the valence band (OFF-state). Under suitable potential, the valence band electron jumped to the conduction band of copper sulfide, and the transport property of the conduction band electrons followed the Ohmic mechanism (ON-state). Fig. 3B, inset, represents the TEM image of the polyanilinestabilized copper sulfide nanoparticles, where the dark spots are the copper sulfide $\left(\mathrm{Cu}_{2} \mathrm{~S}\right)$ nanoparticles within a range of 5-10 $\mathrm{nm}$ dispersed in the polymer matrix.

To determine the endurance property of the device, a pulse train is applied, as mentioned in Fig. 2A (inset), and the device exhibited excellent endurance property for $2 \times 10^{3}$ cycles with an average $\mathrm{ON}-\mathrm{OFF}$ ratio of $\sim 10^{4}$ (Fig. 3C). To extract the retention behaviour (ability of the device to retain its state in the absence of a refreshing power supply) of the device, a pulse

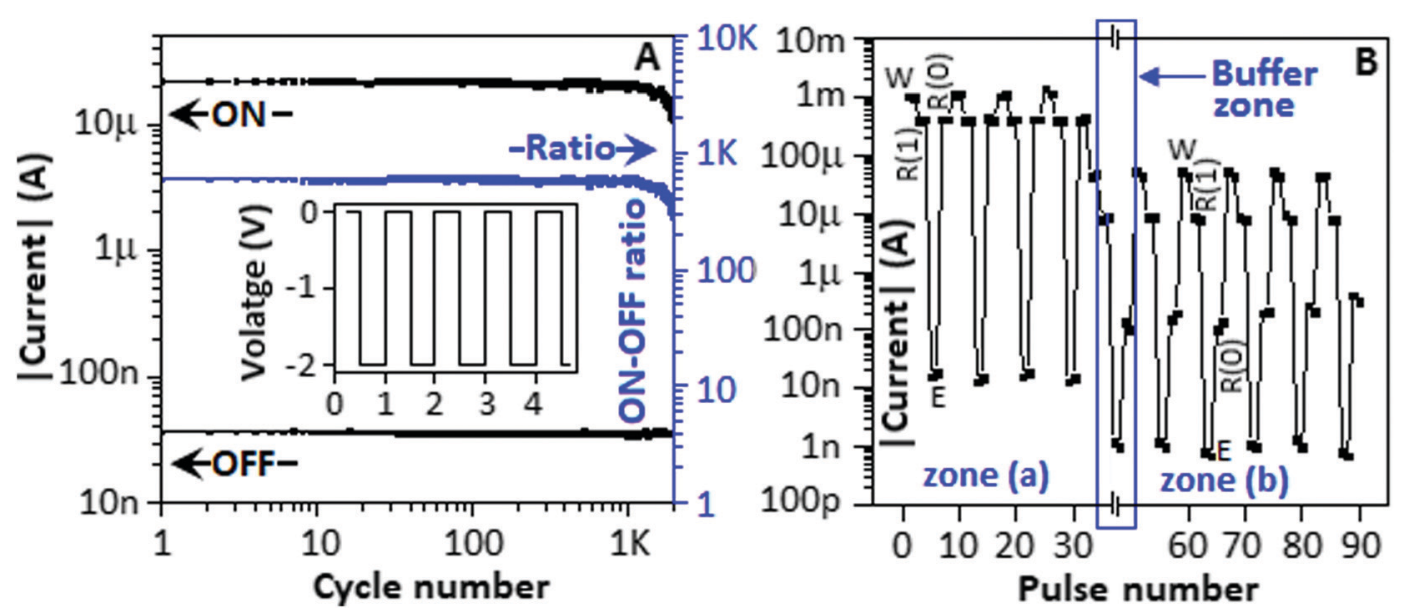

Fig. 2 (A) The endurance study of the device $D(1 b)$ for $2 \times 10^{3}$ cycles. The inset figure shows the pulse train condition for the endurance study. (B) The electrical rewritable property of the device $D$ (I) before and after the addition of cysteine. 

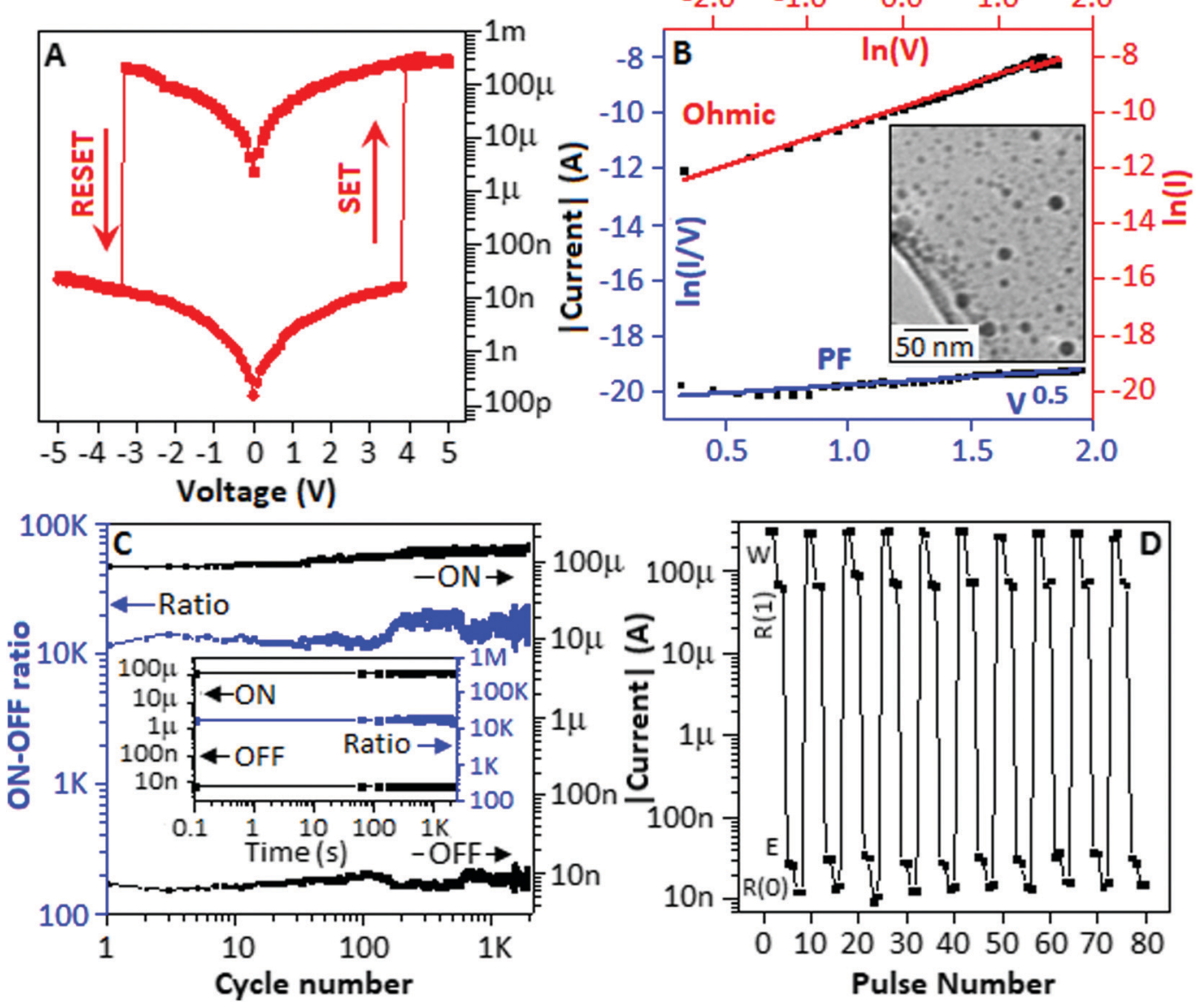

Fig. 3 (A) The current-voltage characteristic of the device Au-PACS-Au, in the semi-log form. (B) The transport behaviour was fitted with the PooleFrenkel (PF) emission and Ohmic conduction mechanism for low and high conductance states, respectively. The inset figure shows the transmission electron microscopy image of polyaniline-stabilized copper sulfide nanoparticles, where the dark spots are the copper sulfide nanoparticles. (C) The endurance study of the device (Au-PACS-Au) for $2 \times 10^{3}$ cycles. Inset figure shows the retention (nonvolatile) study of the Au-PACS-Au device for $2 \times 10^{3} \mathrm{sec}$. (D) The electrical rewritable property of the Au-PACS-Au-based device.

train consisting of $-2 \mathrm{~V}$ for 0.1 sec with a time interval of $60 \mathrm{sec}($ ESI, $\dagger$ Fig. S2) was applied, and the device exhibited an excellent retention capacity for $2 \times 10^{3} \mathrm{sec}$ (Fig. 3C (inset)) with an average $\mathrm{ON}-\mathrm{OFF}$ ratio of $10^{4}$. To study the electrical rewritable property of the device (Au-PACS-Au), the $\mathrm{WR}_{1} \mathrm{ER}_{0}$ cycle is designed, as shown in $\mathrm{ESI} \dagger$ and Fig. S3. The graphical representation (Fig. 3D) of the electrical rewritable property displayed the difference in the current values $(78 \mu \mathrm{A})$ between the $R_{1}$ and $R_{0}$ states. The above study demonstrated that the nonvolatile type of memory was achieved when the stable form of PACS type of the organic-inorganic hybrid system was applied as an active material for the metal-insulator-metal type of device.

In summary, we decoded a chemical complexation process in the language of an electrical signal using an example of the $\mathrm{Cu}(\mathrm{I})$-cysteine system. For the in situ experiment, due to the dynamic nature of the complexation process, the resistive switching performance of the device showed a volatile behaviour, whereas in a stable system (formation of the copper sulfide nanoparticles after the completion of the reaction between $\mathrm{Cu}(\mathrm{I})$ and cysteine), the device displayed a nonvolatile performance with a higher ON-OFF ratio. The proposition of the transport mechanism of a chemical reaction was the main emphasis of this kind of study.

\section{Experimental section}

\section{Device fabrication and electrical characterization}

A glass slide-supported flexible paper was used for the device fabrication, where the role of the slide was to provide the mechanical strength. The active material, $\mathrm{Cu}(\mathrm{I})$-polyaniline/ cysteine/PACS, was dispersed in the form of a thin film with a thickness of $\sim 5 \mu \mathrm{m}$ on the previously deposited bottom electrodes (gold) on the flexible paper. After properly drying, the top gold electrodes were deposited in a crossbar fashion and a sandwich-structured device was fabricated, Au-CuP-Au, D (I)/Au-Cys-Au, D (II)/Au-PACS-Au. The bottom electrode was connected to a high voltage terminal, whereas the top electrode was connected to the ground terminal of the electrical characterization unit. The current-voltage $(I-V)$ measurement was performed at a scan rate of $0.17 \mathrm{~V} \mathrm{~s}^{-1}$ and a step size of 0.1 . 
Keithley 2401 and 2612B source meters were used for the electrical characterization.

Synthesis of polyaniline-stabilized copper sulfide nanoparticles (PACS): in a typical experiment, $0.093 \mathrm{~g}$ of aniline $\left(10^{-2} \mathrm{~mol} \mathrm{dm}^{-3}\right)$ was dissolved in $10 \mathrm{~mL}$ methanol. To this solution, $0.250 \mathrm{~g}$ of $\mathrm{CuSO}_{4} \cdot 5 \mathrm{H}_{2} \mathrm{O}$ in $10 \mathrm{~mL}$ of water $\left(10^{-2} \mathrm{~mol} \mathrm{dm}^{-3}\right)$ was added slowly under continuous stirring conditions. During the addition, the solution appeared green in colour, while at the end, a greenish precipitation was formed, which indicated the formation of the $\mathrm{Cu}(\mathrm{I})$-polyaniline composite at the bottom of the conical flask. To this reaction mixture, $5 \mathrm{~mL}$ of cysteine

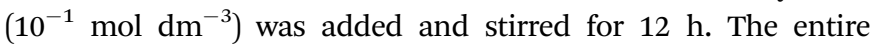
reaction was performed under ambient conditions. The dark brown precipitate material was allowed to settle for $30 \mathrm{~min}$ and collected via a filtration technique. The solid mass was dried under vacuum and applied as an active material for the device (Au-PACS-Au). The X-ray diffraction and X-ray photoelectron spectroscopic characterizations are provided in ESI, $\dagger$ Fig. S4 and S5, respectively.

\section{Conflicts of interest}

There are no conflicts of interest to declare.

\section{Acknowledgements}

This study was financially supported by the Faculty of Science and the Global Excellence and Stature programme, University of Johannesburg.

\section{Notes and references}

1 R. M. Owens and G. G. Malliaras, MRS Bull., 2010, 35, 449-456.

2 Y. Benenson, T. Paz-Elizur, R. Adar, E. Keinan, Z. Livneh and E. Shapiro, Nature, 2001, 414, 430-434.

3 K. y. Tomizaki and H. Mihara, J. Am. Chem. Soc., 2007, 129, 8345-8352.

4 S. Prabhulkar, H. Tian, X. Wang, J. J. Zhu and C. Z. Li, Antioxid. Redox Signaling, 2012, 17, 1796-1822.

5 I. Ron, I. Pecht, M. Sheves and D. Cahen, Acc. Chem. Res., 2010, 43, 945-953.

6 R. Ren, G. Cai, Z. Yu, Y. Zeng and D. Tang, Anal. Chem., 2018, 90, 11099-11105.

7 Z. Luo, Q. Qi, L. Zhang, R. Zeng, L. Su and D. Tang, Anal. Chem., 2019, 91, 4149-4156.

8 F. Meng, L. Jiang, K. Zheng, C. F. Goh, S. Lim, H. H. Hng, J. Ma, F. Boey and X. Chen, Small, 2011, 7, 3016-3020.

9 J. A. Fereiro, X. Yu, I. Pecht, M. Sheves, J. C. Cuevas and D. Cahen, Proc. Natl. Acad. Sci. U. S. A., 2018, 115, E4577-E4583.

10 D. Nandi, A. Taher, R. Ul Islam, S. Siwal, M. Choudhary and K. Mallick, R. Soc. Open Sci., 2016, 3, 160580.
11 D. Nandi, A. Taher, R. U. Isalm and K. Mallick, Appl. Organomet. Chem., 2019, 33, e4733.

12 D. Nandi, S. Siwal and K. Mallick, New J. Chem., 2017, 41, 3082-3088.

13 M. Choudhary, S. K. Shukla, A. Taher, S. Siwal and K. Mallick, ACS Sustainable Chem. Eng., 2014, 2, 2852-2858.

14 H. Tapiero, D. M. Townsend and K. D. Tew, Biomed. Pharmacother., 2003, 57, 386-398.

15 Z. Futera, I. Ide, B. Kayser, K. Garg, X. Jiang, J. H. van Wonderen, J. N. Butt, H. Ishii, I. Pecht, M. Sheves, D. Cahen and J. Blumberger, J. Phys. Chem. Lett., 2020, 11, 9766-9774.

16 K. M. Dokken, J. G. Parsons, J. McClure and J. L. GardeaTorresdey, Inorg. Chim. Acta, 2009, 362, 395-401.

17 L. C. Königsberger, E. Königsberger, G. Hefter and P. M. May, Dalton Trans., 2015, 44, 20413-20425.

18 Y. Peng, L. Shang, Y. Cao, G. I. N. Waterhouse, C. Zhou, T. Bian, L. Z. Wu, C. H. Tung and T. Zhang, Chem. Commun., 2015, 51, 12556-12559.

19 M. Choudhary, R. U. Islam, M. J. Witcomb and K. Mallick, Dalton Trans., 2014, 43, 6396-6405.

20 M. Choudhary, S. Siwal, D. Nandi and K. Mallick, New J. Chem., 2016, 40, 2296-2303.

21 D. Nandi, A. Taher, R. U. Islam, M. Choudhary, S. Siwal and K. Mallick, Sci. Rep., 2016, 6, 33025.

22 R. U. Islam, A. Taher, M. Choudhary, S. Siwal and K. Mallick, Sci. Rep., 2015, 5, 9632.

23 R. U. Islam, A. Taher, M. Choudhary, M. J. Witcomb and K. Mallick, Dalton Trans., 2015, 44, 1341-1349.

24 A. Rigo, A. Corazza, M. L. di Paolo, M. Rossetto, R. Ugolini and M. Scarpa, J. Inorg. Biochem., 2004, 98, 1495-1501.

25 D. Prime and S. Paul, Philos. Trans. R. Soc., A, 2009, 367, 4141-4157.

26 V. K. Perla, S. K. Ghosh, T. Pal and K. Mallick, Physica E, 2020, 116, 113787.

27 V. Bhatt, in Essentials of Coordination Chemistry, ed. V. Bhatt, Academic Press, 2016, ch. 4, pp. 111-137.

28 V. K. Perla, S. K. Ghosh and K. Mallick, ACS Appl. Electron. Mater., 2020, 2, 3987-3993.

29 V. K. Perla, S. K. Ghosh and K. Mallick, Sci. Rep., 2020, 10, 14319.

30 W. Y. Chang, Y. C. Lai, T. B. Wu, S. F. Wang, F. Chen and M. J. Tsai, Appl. Phys. Lett., 2008, 92, 022110.

31 K. H. Chen, K. C. Chang, T. C. Chang, T. M. Tsai, S. P. Liang, T. F. Young, Y. E. Syu and S. M. Sze, Nanoscale Res. Lett., 2016, 11, 224.

32 M. C. Brelle, J. Z. Zhang, L. Nguyen and R. K. Mehra, J. Phys. Chem. A, 1999, 103, 10194-10201.

33 C. Cui, X. Li, J. Liu, Y. Hou, Y. Zhao and G. Zhong, Nanoscale Res. Lett., 2015, 10, 431.

34 V. K. Perla, S. K. Ghosh and K. Mallick, Mater. Adv., 2020, 1, 228-234.

35 V. K. Perla, S. K. Ghosh and K. Mallick, J. Phys. Chem. C, 2021, 125, 1054-1059. 